\title{
Spectral Element Methods for Stochastic Differential Equations with Additive Noise
}

\author{
Chao Zhang *, Dongya Gu and Dongya Tao \\ School of Mathematics and Statistics, Jiangsu Normal University, Xuzhou 221116, \\ P.R. China.
}

Received September 23, 2017; Accepted January 19, 2018

\begin{abstract}
In this paper, we propose numerical schemes for stochastic differential equations driven by white noise and colored noise, respectively. For this purpose, we first discretize the white noise and colored noise, and give their regularity estimates. Then we use spectral element methods to solve the corresponding stochastic differential equations numerically. The approximation errors are derived, and the numerical results demonstrate high accuracy of the proposed schemes.
\end{abstract}

AMS subject classifications: 65M70, 65L60, 41A10, 60H35

Key words: Stochastic differential equations, spectral element methods, high accuracy

\section{Introduction}

Many problems in science and engineering can be characterized by differential equations. However, deterministic differential equations can not reveal the essence of these problems well due to the existence of accidental phenomena and small probability events. Therefore, many scholars consider stochastic differential equations to describe these problems, see $[10,22,23]$ and etc, and the references therein.

Since the solution of a stochastic differential equation (SDE) is a stochastic process, it is difficult to reveal visually the information the process contains. Recently, there are many works to study the numerical solution for stochastic ordinary differential equations (SDEs). Euler-Maruyama method, Milstein method and Runge-Kutta method were proposed to solve SDEs numerically, see $[3,4,13,16,17,19,20,31]$ and the references therein.

On the other hand, many authors investigated the numerical methods for stochastic partial differential equations (SPDEs). Allen et al. [1], Mcdonald [21], Gyöngy [14, 15] used finite difference method to study the numerical solutions of linear SPDEs driven by additive white noise. Also, Walsh [28], Du and Zhang [11] studied finite element method

${ }^{*}$ Corresponding author. Email addresses: chaozhang@jsnu.edu.cn (C. Zhang), gdyjy1@163.com (D.Y. Gu), dongyatao@jsnu.edu.cn (D.Y. Tao) 
for linear SPDEs driven by special noises. Moreover, Cao et al. [6, 7, 8], Theting [26, 27], Yan [29] and etc, have also made some contributions.

As we know, spectral methods take orthogonal polynomials (such as Legendre, Chebyshev, Jacobi, Laguerre and Hermite polynomials) as the basis functions to approximate the solutions of problems in mathematical physics, and tend to have higher accuracy, see $[5,12,25]$. Recently, some scholars are trying to study SPDEs by spectral methods. Shardlow [24] took the eigenvectors of $-\frac{\partial^{2}}{\partial x^{2}}$ subject to Dirichlet boundary condition as the basis functions and used spectral method to approximate the noise. Cao and Yin [9] studied spectral Galerkin method for stochastic wave equations driven by space-time white noise. In addition, Yin and Gan [30] proposed the Chebyshev spectral collocation method to solve a certain type of stochastic delay differential equations.

In the present work, we will try to solve stochastic equations by using spectral element methods. We begin with taking the SDEs driven by white noise into consideration. As we know, the regularity of the solutions of the original stochastic differential equations plays an important role in priori error estimates of the numerical solutions. Unfortunately, the regularity estimates are usually very weak because of the existence of white noise. In order to overcome this difficulty, we will approximate the white noise process by piecewise constant random process as in [1], and apply the Legendre spectral element method for the corresponding stochastic differential equation. We prove the numerical solution converges to the original solution. Moreover, the accuracy of the proposed scheme is indicated by the provided computational results. The other part is that we use spectral element methods to solve stochastic differential equations driven by colored noise numerically. Analogous to the way to deal with white noise, it is nature to use an finite dimensional noise to discretize the colored noise (see [11]), which could improve the regularity of the solutions of the original stochastic differential equations. Therefore, the relevant stochastic differential equation is capable of being approximated by the Legendre spectral element scheme. Furthermore, the numerical results show the high accuracy of spectral element methods.

This paper is organized as follows. In the next section, we study numerical solutions of stochastic differential equations driven by white noise using spectral element methods, and the error estimates as well as the numerical results are presented. In Section 3, we use spectral element methods to solve stochastic differential equations driven by colored noise numerically. The final section is for conclusion remarks.

\section{Numerical solutions of SDEs driven by white noise}

We consider the following stochastic problem driven by white noise

$$
\left\{\begin{array}{l}
-u^{\prime \prime}(x)+b u(x)=g(x)+\dot{W}(x), \quad x \in I:=(0,1), \\
u(0)=u(1)=0,
\end{array}\right.
$$

where $\dot{W}(x)$ denotes white noise, $g(x)$ is a deterministic function and $b$ is a constant. 
The integral form of (2.1) has the form

$$
u(x)+b \int_{0}^{1} k(x, y) u(y) d y=\int_{0}^{1} k(x, y) g(y) d y+\int_{0}^{1} k(x, y) d W(y),
$$

where $k(x, y)=x \wedge y-x y$ is Green's function associated with the elliptic equation

$$
\left\{\begin{array}{l}
-v^{\prime \prime}(x)=\phi(x), \quad x \in I, \\
v(0)=v(1)=0 .
\end{array}\right.
$$

Multiplying the equation (2.1) by $\phi(x)$ and integrating by parts on $I$, we derive that a weak formulation of $(2.1)$ is

$$
\int_{0}^{1} u^{\prime}(x) \phi^{\prime}(x) d x+b \int_{0}^{1} u(x) \phi(x) d x=\int_{0}^{1} g(x) \phi(x) d x+\int_{0}^{1} \phi(x) d W(x),
$$

where $\phi \in C^{2}(0,1) \cap C^{0}[0,1]$.

Remark 2.1. Buckdahn and Pardoux [2] proved existence and uniqueness of solutions to (2.2) and (2.3). Meanwhile, (2.2) and (2.3) are equivalent.

\subsection{Approximation of white noise}

Partition the interval $I$ into $0=x_{0}<x_{1}<\cdots<x_{K}=1$ with $x_{i}=i h, h=x_{i}-x_{i-1}, i=0,1, \cdots, K$. Then a reasonable approximation to white noise on the interval $[0,1]$ is (see [1])

$$
\frac{d \hat{W}(x)}{d x}=\frac{1}{\sqrt{h}} \sum_{i=1}^{K} \eta_{i} \chi_{i}(x),
$$

where

$$
\chi_{i}(x)= \begin{cases}1, & x \in I_{i}:=\left[x_{i-1}, x_{i}\right], \\ 0, & \text { otherwise, }\end{cases}
$$

$\eta_{1}, \eta_{2}, \cdots, \eta_{K}$ are a sequence of random variables that are independent and identically distributed (i.i.d.) and subject to the standard normal distribution $N(0,1)$,

$$
\eta_{i}=\frac{1}{\sqrt{h}} \int_{x_{i-1}}^{x_{i}} d W(x), \quad i=1,2, \cdots, K .
$$

Substituting $d \hat{W}(y)$ for $d W(y)$ in (2.2), we can get

$$
\hat{u}(x)+b \int_{0}^{1} k(x, y) \hat{u}(y) d y=\int_{0}^{1} k(x, y) g(y) d y+\int_{0}^{1} k(x, y) d \hat{W}(y) .
$$

According to Theorem 2.2 in [1], we have the following regularity result. 
Lemma 2.1. Let $\hat{u}$ be the solution of (2.5) with $g \in L^{2}(0,1)$ and $b \geq 0$. Then $\hat{u} \in H^{2}(0,1) \cap H_{0}^{1}(0,1)$ with $E\left(\|\hat{u}\|_{H^{2}}\right) \leq C h^{-\frac{1}{2}}$ where constant $C$ depends only on $g$.

From Theorem 2.1 in [1], we have the estimation between $u$ and $\hat{u}$.

Lemma 2.2. Let $\hat{u}$ be the solution of (2.5), and $u$ be the solution of (2.2). There holds

$$
E\left(\int_{0}^{1}(u(x)-\hat{u}(x))^{2} d x\right) \leq \frac{2 h^{2}}{(1-\lambda)^{2}}
$$

where $\lambda^{2}=b^{2} \int_{0}^{1} \int_{0}^{1} k^{2}(x, y) d x d y<1$

Remark 2.2. Lemma 2.2 implies that if the mesh is sufficiently fine, then $\hat{u}(x)$ is a good approximation to $u(x)$, and $\hat{u}(x)$ is smoother than $u(x)$.

\subsection{Spectral element methods for stochastic differential problem (2.1)}

Denote $H_{0}^{1}(I)=\left\{u \mid u, u^{\prime} \in L^{2}(I), u(0)=u(1)=0\right\}$, we substitute $\hat{W}(x)$ for $W(x)$, then the weak form (2.3) of the problem (2.1) can be written as

$$
\begin{aligned}
& \int_{0}^{1} \hat{u}^{\prime}(x) \phi^{\prime}(x) d x+b \int_{0}^{1} \hat{u}(x) \phi(x) d x \\
= & \int_{0}^{1} g(x) \phi(x) d x+\int_{0}^{1} \phi(x) d \hat{W}(x), \quad \forall \phi \in H_{0}^{1}(I) .
\end{aligned}
$$

Let

$$
X^{K, N}=\left\{u \in C^{0}(I)|u|_{I_{i}} \in P_{N}\right\}, \quad X_{0}^{K, N}=\left\{u \in X^{K, N} \mid u(0)=u(1)=0\right\},
$$

where $P_{N}$ be the space of the algebraic polynomials with degree at most $N$. Then the corresponding Galerkin scheme of (2.7) is to find $u_{N}\left(\in X_{0}^{K, N}\right)$, such that

$$
\begin{aligned}
& \int_{0}^{1} u_{N}^{\prime}(x) \phi^{\prime}(x) d x+b \int_{0}^{1} u_{N}(x) \phi(x) d x \\
= & \int_{0}^{1} g(x) \phi(x) d x+\int_{0}^{1} \phi(x) d \hat{W}(x), \quad \forall \phi \in X_{0}^{K, N} .
\end{aligned}
$$

Define the Sobolev space:

$$
B_{0,0}^{m}(I)=\left\{u: \int_{0}^{1}\left(\partial_{x}^{k} u(x)\right)^{2} x^{k}(1-x)^{k} d x<\infty, 0 \leq k \leq m\right\}, \quad m \in \mathbb{N} .
$$

By using Theorem 3.39 in [25], we obtain the following result on I directly. 
Lemma 2.3. If $\hat{u} \in H_{0}^{1}(I), \partial_{x} \hat{u} \in B_{0,0}^{m-1}(I)$, and $\partial_{x}^{m} \hat{u} \in L^{2}(I)$, then for $1 \leq m \leq N+1$ and $\mu=0,1$,

$$
\left\|\hat{u}-u_{N}\right\|_{\mu, I} \leq C h^{m-\mu} N^{\mu-m}\left\|\partial_{x}^{m} \hat{u}\right\|_{I},
$$

where $C$ is a positive constant independent of $\hat{u}, h, m$ and $N$.

Remark 2.3. In particular, for $m=2$, let $\hat{u}$ and $u_{N}$ be the solutions of (2.7) and (2.8), we have

$$
\left\|\hat{u}-u_{N}\right\|_{I} \leq C h^{2} N^{-2}\left\|\partial_{x}^{2} \hat{u}\right\|_{I}
$$

Therefore, by the triangle inequality, Lemma 2.2, (2.9) and Lemma 2.1, successively, we have the following error estimates.

Theorem 2.1. Let $u$ and $u_{N}$ be the solutions of (2.1) and (2.8), respectively. Then we have

$$
E\left(\left\|u-u_{N}\right\|_{L^{2}, I}\right) \leq C\left(\frac{h}{1-\lambda}+h^{\frac{3}{2}} N^{-2}\right)
$$

where $C$ is a positive constant independent of $h$ and $N$.

\subsection{Numerical results}

Let

$$
\begin{aligned}
& \phi_{0}(\xi)=\frac{1-\xi}{2}, \quad \phi_{N}(\xi)=\frac{1+\xi}{2}, \\
& \phi_{m}(\xi)=L_{m-1}(\xi)-L_{m+1}(\xi), \quad 1 \leq m \leq N-1, \quad \xi \in(-1,1) .
\end{aligned}
$$

Here, $L_{m}(\xi)$ is the Legendre polynomial on $(-1,1)$ with degree $m$, which satisfy that $L_{m}( \pm 1)=( \pm 1)^{m}$, and fulfill the following relations.

$$
\begin{gathered}
\int_{-1}^{1} L_{m}(\xi) L_{l}(\xi) d x=\gamma_{m} \delta_{m l}, \quad \gamma_{m}=\frac{2}{2 m+1}, \\
(2 m+1) L_{m}(\xi)=L_{m+1}^{\prime}(\xi)-L_{m-1}^{\prime}(\xi), \quad m \geq 1 .
\end{gathered}
$$

In actual computation, we introduce the boundary-inner decomposition basis

$$
\left\{\phi_{m}(\xi)\right\}_{m=0}^{N}=\left\{\phi_{0}(\xi), \phi_{N}(\xi)\right\} \cup\left\{\phi_{m}(\xi), \phi_{m}( \pm 1)=0\right\}_{m=1}^{N-1}
$$

We expand them into global variable by

$$
\psi_{m}^{(i)}(x)= \begin{cases}\phi_{m}(\xi), & \text { if } x \in I_{i}, \quad \xi=m_{i}^{-1}(x), \\ 0, & \text { if } x \notin I_{i},\end{cases}
$$


where $m_{i}(\xi)=x_{i-1} \phi_{0}(\xi)+x_{i} \phi_{N}(\xi), i=1, \cdots, K, m=1, \cdots, N-1$. For ensuring the continuity of the solution of the problem, we must propose the basis functions at interfaces. Therefore, we introduce

$$
\psi_{i}^{*}(x)= \begin{cases}\psi_{N}^{(i)}(x), & x \in I_{i}, \\ \psi_{0}^{(i+1)}(x), & x \in I_{i+1}, \\ 0, & \text { otherwise }\end{cases}
$$

where $i=1, \cdots, K-1$. At the left and right endpoints, we define

$$
\begin{aligned}
& \psi_{0}^{*}(x)= \begin{cases}\psi_{0}^{(1)}(x), & x \in I_{1}, \\
0, & \text { otherwise, }\end{cases} \\
& \psi_{K}^{*}(x)= \begin{cases}\psi_{N}^{(K)}(x), & x \in I_{K}, \\
0, & \text { otherwise. }\end{cases}
\end{aligned}
$$

So the global basis set are

$$
V=\left\{\psi_{j}(x)\right\}_{j=0}^{K N}:=\left\{\psi_{0}^{*}(x), \psi_{K}^{*}(x)\right\} \cup\left\{\psi_{i}^{*}(x)\right\}_{i=1}^{K-1} \cup\left\{\psi_{m}^{(i)}(x), m=1, \cdots, N-1\right\}_{i=1}^{K} .
$$

Then, we expand the numerical solution of the problem (2.1) as follows:

$$
u_{N}(x)=\sum_{j=0}^{K N} \tilde{u}_{j} \psi_{j}(x) .
$$

Substituting the above expression into Galerkin scheme (2.8) and taking $\phi=\psi_{l}(x), l=$ $0, \cdots, K N$, which yields that

$$
\begin{aligned}
& \sum_{j=0}^{K N} \tilde{u}_{j} \int_{0}^{1} \psi_{j}^{\prime}(x) \psi_{l}^{\prime}(x) d x+b \sum_{j=0}^{K N} \tilde{u}_{j} \int_{0}^{1} \psi_{j}(x) \psi_{l}(x) d x \\
= & \int_{0}^{1} g(x) \psi_{l}(x) d x+\int_{0}^{1} \psi_{l}(x) d \hat{W}(x), \quad l=0, \cdots, K N .
\end{aligned}
$$

Let us denote

$$
\begin{array}{lc}
M_{l, j}=\int_{0}^{1} \psi_{j}(x) \psi_{l}(x) d x, & \boldsymbol{M}=\left(M_{l, j}\right)_{0 \leq j, l \leq K N}, \\
S_{l, j}=\int_{0}^{1} \psi_{j}^{\prime}(x) \psi_{l}^{\prime}(x) d x, & \boldsymbol{S}=\left(S_{l, j}\right)_{0 \leq j, l \leq K N}, \\
\hat{f}_{l}=\int_{0}^{1} g(x) \psi_{l}(x) d x+\int_{0}^{1} \psi_{l}(x) d \hat{W}(x), \\
\boldsymbol{F}=\left(\hat{f}_{0}, \hat{f}_{1}, \cdots, \hat{f}_{K N}\right)^{t}, \quad \boldsymbol{U}=\left(\tilde{u}_{0}, \tilde{u}_{1}, \cdots, \tilde{u}_{K N}\right)^{t} .
\end{array}
$$

Then the linear algebraic system (2.19) becomes the following compact form

$$
(S+b \boldsymbol{M}) \boldsymbol{U}=\boldsymbol{F} .
$$


To compute the error $E\left\|u-u_{N}\right\|_{L^{2}}$, we set the state of random numbers to be 400 , perform 10000 runs with different samples of noise for each computation, then for each sample calculate $\left\|u-u_{N}\right\|_{L^{2}}$, and finally, obtain the average value of $\left\|u-u_{N}\right\|_{L^{2}}$.

For the problem (2.1) with $b=\frac{1}{2}$, we take the test function $u(x)=x(1-x) \sin 3 x$. In Table 1, the values of the error $E\left\|u-u_{N}\right\|_{L^{2}}$ with various $N$ and $K$ are listed. We can see that the errors decay as $N$ and $K$ increase. Comparison with the numerical results in [1], Allen, Novosel and Zhang took $h=0.0294$ and the smoother test function $u(x)=x(1-x)$, the accuracy of errors can only be reached to $O\left(10^{-4}\right)$. While in Table 1 , though the test function $u(x)=x(1-x) \sin 3 x$ is oscillatory, the accuracy can be reached to $O\left(10^{-6}\right)$ with $N=8$. This indicates that our new approach seems to be more accurate.

Table 1: The values of $E\left\|u_{N}-u\right\|_{L^{2}}$.

\begin{tabular}{||c|c|c|c||}
\hline & $K=1$ & $K=2$ & $K=3$ \\
\hline$N=4$ & $5.03 e-05$ & $4.19 e-06$ & $5.55 e-06$ \\
$N=6$ & $3.59 e-06$ & $4.72 e-06$ & $6.30 e-06$ \\
$N=8$ & $4.05 e-06$ & $5.24 e-06$ & $6.97 e-06$ \\
\hline
\end{tabular}

\section{Numerical solutions of SDEs driven by colored noise}

Due to the existence of white noise, the solution to (2.1) has low regularity, thus it doesn't achieve higher precision, which can be seen from Table 1. In this section, we will study numerical methods for stochastic differential equations driven by colored noise.

\subsection{Properties of colored noise}

Let $W=\{W(t), t \geq 0\}$ be a standard Wiener process and for fixed $h>0$, we define colored noise by (see [18])

$$
\dot{W}(x)=\frac{W(x+h)-W(x)}{h}, \quad \forall x \geq 0 .
$$

This is a wide-sense stationary Gaussian process with zero means and with covariances

$$
c(x-s)=\frac{1}{h} \max \left\{0,1-\frac{1}{h}|x-s|\right\},
$$

it thus has spectral density

$$
S_{h}(v)=\frac{1}{h} \int_{-h}^{h}\left(1-\frac{|s|}{h}\right) \cos (2 \pi v s) d s=\left(\frac{\sin (2 \pi v h)}{\pi v h}\right)^{2} .
$$

In general, we may use the following abstract formulation to simulate colored noise (see [11]):

$$
\dot{W}(x)=\sum_{k=1}^{\infty} \sigma_{k} \eta_{k} \varphi_{k}(x),
$$


where the i.i.d. random sequence $\eta_{k} \sim N(0,1)$, the deterministic functions $\left\{\varphi_{k}(x)\right\}$ form an orthogonal basis of $L^{2}(I)$ or its subspace, and the coefficients $\left\{\sigma_{k}\right\}$ are to be chosen to ascertain the convergence of the series in the mean square sense with respect to some suitable norms.

Let $\left\{\sigma_{k}^{n}\right\}_{k=1}^{\infty}$ approach $\left\{\sigma_{k}\right\}_{k=1}^{\infty}$ as $n \rightarrow \infty$ in some appropriate sense, then an approximation of $\dot{W}(x)$ is

$$
\dot{W}_{n}(x)=\sum_{k=1}^{\infty} \sigma_{k}^{n} \eta_{k} \varphi_{k}(x),
$$

where

$$
\sigma_{k}^{n}= \begin{cases}\sigma_{k}, & k \leq n, \\ 0, & k>n .\end{cases}
$$

As was shown by Du and Zhang [11], the faster the coefficients $\sigma_{k}$ decay, the smoother the noise trajectory $\dot{W}_{n}(x)$ looks. On the other hand, if the coefficients decay sufficiently slowly, then the trajectory can clearly resemble that of a white noise away from the boundary. In the end of this section, we will give the numerical results to demonstrate that different forms of coefficients $\sigma_{k}^{n}$ induce different rates of convergence.

From Lemma 3.1 in [11], a bound is stated on $\dot{W}_{n}$ in the following lemma.

Lemma 3.1. For $\dot{W}_{n}(x)=\sum_{k=1}^{\infty} \sigma_{k}^{n} \eta_{k} \varphi_{k}(x), \varphi_{k}(x)=\sqrt{2} \sin k \pi x$, if $s$ is a non-negative integer, then

$$
E\left\|\dot{W}_{n}\right\|_{H^{s}} \leq C\left(\sum_{k=1}^{\infty}\left(\sigma_{k}^{n} k^{s}\right)^{2}\right)^{\frac{1}{2}}
$$

provided that the right-hand side is convergent.

\subsection{Spectral element methods for SDEs driven by colored noise}

We consider the following stochastic problem driven by colored noise

$$
\left\{\begin{array}{l}
-u^{\prime \prime}(x)+b u(x)=g(x)+\dot{W}(x), \quad x \in I, \\
u(0)=u(1)=0,
\end{array}\right.
$$

where $\dot{W}(x)$ denotes colored noise, $g(x)$ is a deterministic function and $b$ is a constant.

The integral form of the problem (3.4) is

$$
u(x)+b \int_{0}^{1} k(x, y) u(y) d y=\int_{0}^{1} k(x, y) g(y) d y+\int_{0}^{1} k(x, y) d W(y),
$$

and the weak form is

$$
\begin{aligned}
& \int_{0}^{1} u^{\prime}(x) \phi^{\prime}(x) d x+b \int_{0}^{1} u(x) \phi(x) d x \\
= & \int_{0}^{1} g(x) \phi(x) d x+\int_{0}^{1} \phi(x) d W(x), \quad \forall \phi \in C^{2}(0,1) \cap C^{0}[0,1] .
\end{aligned}
$$


We replace $d W(y)$ with $d W_{n}(y)$ in (3.5), and obtain

$$
\hat{u}(x)+b \int_{0}^{1} k(x, y) \hat{u}(y) d y=\int_{0}^{1} k(x, y) g(y) d y+\int_{0}^{1} k(x, y) d W_{n}(y) .
$$

According to Theorem 3.1 in [11], the following lemma holds.

Lemma 3.2. For $\dot{W}_{n}(x)=\sum_{k=1}^{\infty} \sigma_{k}^{n} \eta_{k} \varphi_{k}(x), \varphi_{k}(x)=\sqrt{2} \sin k \pi x$, if $u$ and $\hat{u}$ are the solutions of (3.5) and (3.7), respectively, then, for some constant $C>0$,

$$
E\|u-\hat{u}\|_{L^{2}, I} \leq \frac{C}{1-\lambda}\left\|\vec{\sigma}^{n}-\vec{\sigma}\right\|_{Q_{-1}}
$$

where $\lambda^{2}=b^{2} \int_{0}^{1} \int_{0}^{1} k^{2}(x, y) d x d y<1, \vec{\sigma}^{n}=\left(\sigma_{1}^{n}, \sigma_{2}^{n}, \cdots, \sigma_{k}^{n}, \cdots\right)^{t}, \vec{\sigma}=\left(\sigma_{1}, \sigma_{2}, \cdots, \sigma_{k}, \cdots\right)^{t}$,

$$
\left\|\vec{\sigma}^{n}-\vec{\sigma}\right\|_{Q_{-1}}^{2}=\sum_{k=1}^{\infty} \sum_{l=1}^{\infty}\left(\sigma_{k}^{n}-\sigma_{k}\right)\left(\sigma_{l}^{n}-\sigma_{l}\right)(k l)^{-1} \delta_{k l}
$$

Substituting $W_{n}(x)$ for $W(x)$ in (3.6), the weak form (3.6) can be written as

$$
\begin{aligned}
& \int_{0}^{1} \hat{u}^{\prime}(x) \phi^{\prime}(x) d x+b \int_{0}^{1} \hat{u}(x) \phi(x) d x \\
= & \int_{0}^{1} g(x) \phi(x) d x+\int_{0}^{1} \phi(x) d W_{n}(x), \quad \forall \phi(x) \in H_{0}^{1}(I),
\end{aligned}
$$

and the corresponding Galerkin scheme of (3.4) is to find $u_{N}\left(\in X_{0}^{K, N}\right)$, such that

$$
\begin{aligned}
& \int_{0}^{1} u_{N}^{\prime}(x) \phi^{\prime}(x) d x+b \int_{0}^{1} u_{N}(x) \phi(x) d x \\
= & \int_{0}^{1} g(x) \phi(x) d x+\int_{0}^{1} \phi(x) d W_{n}(x), \quad \forall \phi \in X_{0}^{K, N} .
\end{aligned}
$$

Following the same line of Theorem 2.1, together with the triangle inequality, Lemma 3.2 and Lemma 2.3, we then have the following error estimates.

Theorem 3.1. Let $u$ and $u_{N}$ are the solutions of (3.4) and (3.10), respectively. Then we have

$$
E\left(\left\|u-u_{N}\right\|_{L^{2}, I}\right) \leq C\left(\left\|\vec{\sigma}^{n}-\vec{\sigma}\right\|_{Q_{-1}}+h^{m} N^{-m}\left\|\partial_{x}^{m} \hat{u}\right\|_{I}\right),
$$

where $C$ is a positive constant independent of $u, h, m$ and $N$. Furthermore, according to Lemma 3.1 , for $2 \leq m \leq N+1$, we have

$$
E\left(\left\|u-u_{N}\right\|_{L^{2}, I}\right) \leq C\left(\left\|\vec{\sigma}^{n}-\vec{\sigma}\right\|_{Q_{-1}}+h^{m} N^{-m}\left(\sum_{k=1}^{\infty}\left(\sigma_{k}^{n} k^{m-2}\right)^{2}\right)^{\frac{1}{2}}\right) .
$$




\subsection{Numerical results}

In actual computation, we expand the numerical solution of the problem (3.4) as follows:

$$
u_{N}(x)=\sum_{j=0}^{K N} \hat{u}_{j} \psi_{j}(x),
$$

where $\left\{\psi_{j}\right\}_{j=0}^{K N}$ are defined in (2.14)-(2.17). Substituting the above expansion into Galerkin scheme (3.10) and taking $\phi=\psi_{l}(x), l=0, \cdots, K N$, we can obtain the following system of linear algebraic equations

$$
\begin{aligned}
& \sum_{j=0}^{K N} \hat{u}_{j} \int_{0}^{1} \psi_{j}^{\prime}(x) \psi_{l}^{\prime}(x) d x+b \sum_{j=0}^{K N} \hat{u}_{j} \int_{0}^{1} \psi_{j}(x) \psi_{l}(x) d x \\
= & \int_{0}^{1} g(x) \psi_{l}(x) d x+\int_{0}^{1} \psi_{l}(x) d W_{n}(x), \quad l=0, \cdots, K N .
\end{aligned}
$$

Let us denote

$$
\begin{aligned}
& \hat{f}_{l}=\int_{0}^{1} g(x) \psi_{l}(x) d x+\int_{0}^{1} \psi_{l}(x) d W_{n}(x), \\
& \boldsymbol{F}=\left(\hat{f}_{0}, \hat{f}_{1}, \cdots, \hat{f}_{K N}\right)^{t}, \quad \boldsymbol{U}=\left(\hat{u}_{0}, \hat{u}_{1}, \cdots, \hat{u}_{K N}\right)^{t} .
\end{aligned}
$$

Then we can rewrite the linear algebraic system (3.13) as

$$
(S+b \boldsymbol{M}) \boldsymbol{U}=\boldsymbol{F},
$$

where the matrix $S, M$ is defined as in (2.20).

For numerical experiment, we let $b=\frac{1}{2}$, and the test function $u_{d}(x)=x(1-x) \sin 3 x$. Then the exact solution to (3.4) is given by $u(x)=u_{d}(x)+u_{s}(x)$, where

$$
u_{s}(x)=\sum_{k=1}^{\infty} \frac{\sqrt{2} \sigma_{k}}{b+(k \pi)^{2}} \eta_{k} \sin k \pi x .
$$

The state of random numbers is also set to be 400. Tables 2-3 list the values of the error $E\left\|u-u_{N}\right\|_{L^{2}}$ with $\sigma_{k}=k^{-\frac{3}{2}}, k^{-\frac{7}{2}}$, respectively. The results indicate that the errors decay rapidly as $N$ and $K$ increase. At the same time, they demonstrate that if the coefficients $\sigma_{k}$ decay sufficiently fast, the numerical solution would be smoother.

Also, in [11], Du and Zhang took $h=\frac{1}{128}$ and the smoother test function $u_{d}(x)=x(1-$ $x)$, the accuracy of error estimates could only be reached to $O\left(10^{-6}\right)$ when $\sigma_{k}=k^{-\frac{3}{2}}$. While for spectral element methods, from Table 2, the accuracy of error estimates can be reached to $O\left(10^{-13}\right)$ with $N=240$, even if we take the oscillatory function as $u_{d}(x)=x(1-x) \sin 3 x$. For $\sigma_{k}=k^{-\frac{7}{2}}$, took the same $h$ and test function, Du and Zhang [11] get the accuracy of estimates reached to $O\left(10^{-10}\right)$. As we can see from Table 3, the accuracy can be reached to $O\left(10^{-14}\right)$ only with $N=140$. This demonstrates the accuracy of the proposed method. 
Table 2: The values of $E\left\|u_{N}-u\right\|_{L^{2}}$ with $\sigma_{k}=k^{-\frac{3}{2}}$.

\begin{tabular}{||c|c|c|c||}
\hline & $K=1$ & $K=2$ & $K=3$ \\
\hline$N=20$ & $3.97 e-05$ & $2.10 e-05$ & $1.41 e-05$ \\
$N=60$ & $1.33 e-05$ & $6.71 e-06$ & $4.35 e-06$ \\
$N=100$ & $7.91 e-06$ & $3.73 e-06$ & $2.37 e-06$ \\
$N=140$ & $5.61 e-06$ & $2.56 e-06$ & $2.62 e-09$ \\
$N=180$ & $4.26 e-06$ & $1.62 e-06$ & $1.48 e-10$ \\
$N=220$ & $3.39 e-06$ & $9.54 e-10$ & $2.21 e-11$ \\
$N=240$ & $2.83 e-06$ & $2.02 e-10$ & $2.32 e-13$ \\
\hline
\end{tabular}

Table 3: The values of $E\left\|u_{N}-u\right\|_{L^{2}}$ with $\sigma_{k}=k^{-\frac{7}{2}}$.

\begin{tabular}{||c|c|c|c||}
\hline & $K=1$ & $K=2$ & $K=3$ \\
\hline$N=20$ & $4.95 e-08$ & $6.13 e-09$ & $1.82 e-09$ \\
$N=60$ & $1.87 e-09$ & $2.27 e-10$ & $6.71 e-11$ \\
$N=100$ & $4.08 e-10$ & $4.87 e-11$ & $1.44 e-11$ \\
$N=140$ & $1.50 e-10$ & $1.77 e-11$ & $1.12 e-14$ \\
\hline
\end{tabular}

\section{Conclusion}

In this paper, the Legendre spectral element schemes were proposed for stochastic differential equations driven by white noise and colored noise, respectively. For stochastic differential equations driven by white noise, we improved the regularity of the solution by employing piecewise constant random process to approximate white noise process. Thus, the Legendre spectral element scheme was able to apply to approximate the corresponding stochastic differential equation. The error analysis was provided and the accuracy of the proposed scheme was showed by the numerical experiments. As for stochastic differential equations driven by colored noise, we approximated colored noise process by a finite dimensional noise and employed the Legendre spectral element scheme to the corresponding stochastic differential equation. The error estimation was presented and the numerical results demonstrated the high accuracy of the proposed schemes.

Although the spectral element methods was only considered for second order elliptic stochastic differential equations in the present work, it is suitable to other types of stochastic differential equations.

\section{Acknowledgments}

This work is supported in part by NSF of China No. 11571151 and No. 11771299, and Priority Academic Program Development of Jiangsu Higher Education Institutions. 


\section{References}

[1] E. J. Allen, S. J. Novosel and Z. M. Zhang, Finite element and difference approximation of some linear stochastic partial differential equations, Stochastics Stochastics Rep., 64(12)(1998), 117-142.

[2] R. Buckdahn and E. Pardoux, Monotonicity methods for white noise driven quasi-linear SPDEs, Diffusion Processes and related Problems in Analysis., Vol.1, 1990.

[3] K. Burrage and P. M. Burrage, High strong order explicit Runge-Kutta methods for stochastic ordinary differential equations, Appl. Numer. Math., 22(1996), 81-101.

[4] K. Burrage, P. M. Burrage and T. Tian, Numerical methods for strong solutions of stochastic differential equations: An overview, Proc. R. Soc. A: Math. Phys. Eng. Sci., 460(2004), 373402.

[5] C. Canuto, M. Y. Hussaini, A. Quarteroni and T. A. Zang, Spectral methods: evolution to complex geometries and applications to fluid dynamics, Springer-Verlag, Berlin, 2007.

[6] Y. Cao, Finite element and discontinuous Galerkin method for stochastic helmholtz equation in two-and-three dimensions, J. Comput. Math., 26(5)(2008), 702-715.

[7] Y. Cao, Z. Chen and M. Gunzburger, Error analysis of finite element approximations of the stochastic Stokes equations, Adv. Comput. Math., 33(2)(2010), 215-230.

[8] Y. Cao, H. T. Yang and L. Yan, Finite element methods for semilinear elliptic stochastic partial differential equations, Numer. Math., 106(2007), 181-198.

[9] Y. Cao and L. Yin, Spectral Galerkin method for stochastic wave equations driven by spacetime white noise, Commun. Pure Appl. Anal., 6(3)(2007), 607-617.

[10] E. Cinlae, Introduction to stochastic processes, Englewood cliffs, New Jersey: Prentice-hall, Inc., 1975.

[11] Q. Du and T. Zhang, Numerical approximation of some linear stochastic partial differential equations driven by special additive noise, SIAM. J. Numer. Anal., 40(2002), 1421-1445.

[12] B. Y. Guo, Spectral methods and their applications, World Scientific Publishing Co. Inc., River Edge, NJ, 1998.

[13] Q. Guo, W. Liu, X. Mao and R. Yue, The truncated milstein method for stochastic differential equations, arXiv: 1704. 04135v2., 2017.

[14] I. Gyöngy, Lattice approximations for stochastic quasi-linear parabolic partial differential equations driven by space-time white noise, I, Potential Anal., 9(1998), 1-25.

[15] I. Gyöngy, Lattice approximations for stochastic quasi-linear parabolic partial differential equations driven by space-time white noise, II, Potential Anal., 11(1999), 1-37.

[16] D. J. Higham, X. Mao and A. M. Stuart, Strong convergence of Euler-like methods for nonlinear stochastic differential equations, SIAM J. Numer. Anal., 40(2002), 1041-1063.

[17] D. J. Higham, X. Mao and A. M. Stuart, Exponential mean square stability of numerical solutions to stochastic differential equations, London Mathematical Society J. Comput. Math., 6(2003), 297-313.

[18] P. E. Kloedn and E. Platen, Numerical solution of stochastic differential equations, SpringerVerlag Berlin Heidelberg., 1999.

[19] X. Mao, The truncated Euler-Maruyama method for stochastic differential equations, J.Comput. Appl. Math., 290(2015), 370-384.

[20] N. Mariko and N. Syoiti, A new high-order weak approximation scheme for stochastic differential equations and the Runge-Kutta method, Finance Stoch., 13(2009), 415-443.

[21] S. Mcdonald, Finite difference approximation for linear stochastic partial differential equations with method of lines, Munich Personal RePEc Archive, 2006. 
[22] B. Øksendal, Stochastic differential equations. Springer-Verlag Berlin Heidelberg, 2005.

[23] L. R. Rabiner, A tutorial on hidden Markov models and selected applications in speech secognition, Proceedings of IEEE.,77(2)(1989), 257-286.

[24] T. Shardlow, Numerical methods for stochastic parabolic PDEs. Numer. Funct. Anal. Optim., 20(1999), 121-145.

[25] J. Shen, T. Tang and L. L. Wang, Spectral methods: algorithms, analysis and applications, volume 41 of Series in Computational Mathematics. Springer-Verlag, Berlin, Heidelberg., 2011.

[26] T. G. Theting, Solving Wick-stochastic boundary value problems using a finite element method. Stochastics Stochastics Rep., 70(3-4)(2000), 241-270.

[27] T. G. Theting, Solving parabolic Wick-stochastic boundary value problems using a finite element method, Stochastics Stochastics Rep., 75(1-2)(2003), 49-77.

[28] J. B. Walsh, Finite element methods for parabolic stochastic PDES, Poten. Anal., 23(2005), 1-43.

[29] Y. Yan, Semidiscrete Galerkin approximation for a linear stochastic parabolic partial differential equation driven by an additive noise, BIT. Numer. Math., 4(2004), 829-847.

[30] Z. W. Yin and S. Q. Gan, Chebyshev spectral collocation method for stochastic delay differential equations, Adv. Difference Equ., 1(2015), 1-12.

[31] R. Zeghdane, L. Abbaoui and A. Tocino., Higher-order semi-implicit Taylor schemes for Itö stochastic differential equations, J. Comput. Appl. Math., 236(2011), 1009-1023. 